\title{
BMJ Open Prognostic effects of health-related quality of life at baseline and early change in health-related quality of life on response to treatment and survival in patients with advanced lung cancer: a prospective observational study in China
}

\author{
Jiali Liu (10), ${ }^{1}$ Yuxiang Ma, ${ }^{2}$ Ruizhen Gao, ${ }^{3}$ Xia Liu, ${ }^{3}$ Yalan Wang, ${ }^{3}$ Juan Yu, \\ Jianhua Zhan, ${ }^{2}$ Yan Huang, ${ }^{3}$ Huiyin Qin, ${ }^{1}$ Li Zhang ${ }^{3}$
}

To cite: Liu J, Ma Y, Gao R, et al. Prognostic effects of health-related quality of life at baseline and early change in health-related quality of life on response to treatment and survival in patients with advanced lung cancer: a prospective observational study in China. BMJ Open 2022;12:e047611. doi:10.1136/ bmjopen-2020-047611

- Prepublication history for this paper is available online To view these files, please visit the journal online (http://dx.doi org/10.1136/bmjopen-2020047611).

$\mathrm{JL}, \mathrm{YM}$ and $\mathrm{RG}$ are joint first authors.

Received 04 December 2020 Accepted 14 December 2021

Check for updates

(c) Author(s) (or their employer(s)) 2022. Re-use permitted under CC BY-NC. No commercial re-use. See rights and permissions. Published by BMJ.

For numbered affiliations see end of article.

Correspondence to

Professor Li Zhang;

zhangli6@mail.sysu.edu.cn

\section{ABSTRACT}

Objectives To investigate the relationship among baseline health-related quality of life (HRQoL), early changes in $\mathrm{HRQOL}$ from baseline to completion of the first cycle of chemotherapy, and prognosis in patients with advanced lung cancer.

Design This was a prospective, observational study. Setting The study was conducted in a national cancer centre in South China.

Participants A total of 243 patients with chemo-naïve with advanced lung cancer were enrolled.

Intervention None.

Primary and secondary outcome measures The Functional Assessment of Cancer Therapy-Lung was used to assess HRQOL at baseline and at the end of the first cycle of chemotherapy. The Trial Outcome Index (TOI) and Lung Cancer Scale (LCS) were calculated as predictive indicators. Response to treatment was evaluated as per the Response Evaluation Criteria in Solid Tumors (RECIST) V.1.1. Survival data were gathered from follow-up to September 2019.

Results Patients with 5-point or greater decreases in T0I (65\% vs $48 \%$, adjusted risk ratio $(\mathrm{aRR})=2.19,95 \% \mathrm{Cl} 1.09$ to 4.41$)$ or 2-point or greater decreases in LCS $(72 \%$ vs $48 \%, a R R=3.29,95 \% \mathrm{Cl} 1.50$ to 7.22 ) from baseline to completion of the first cycle of chemotherapy were more likely to show stable or progressive disease than those whose HRQoL had improved. Baseline TOI $\leq 54(80.0 \%$ vs $69.9 \%$, adjusted hazard risk $(\mathrm{aHR})=1.36,95 \% \mathrm{Cl} 1.01$ to 1.84) and $\mathrm{LCS} \leq 21(77.6 \%$ vs $72.5 \%$, aHR $=1.36,95 \% \mathrm{Cl}$ 1.01 to 1.83) were associated with higher risk for death compared with TOl>54 and LCS $>21$. Area under the curve analysis indicated that early changes in LCS and baseline LCS scores could better predict response to treatment and overall survival than the corresponding TOI values. Conclusions Higher pretreatment HRQoL scores could predict longer survival, while declining HRQOL values could predict unfavourable treatment outcome among patients with advanced lung cancer. The use of the LCS is
Strengths and limitations of this study

This is the first cohort established to explore the relationship between early changes in health-related quality of life and prognosis in patients with advanced lung cancer in China.

- Early changes in quality of life were used as predictors for response to treatment and overall survival for patients with lung cancer, which may supplement current prognostic predictors.

- Clinically meaningful thresholds for early changes in health-related quality of life with the potential to serve as references for clinical practice were explored.

- Real-world data were collected in this study, thus complementing those obtained through the analysis of secondary data from clinical trials.

- Only patients who received chemotherapy were recruited in this study, which may have compromised the representativeness of the sample.

recommended for the routine collection of patient-reported HRQOL.

Trial registration number NCT01914120.

\section{INTRODUCTION}

Lung cancer is the second most frequently diagnosed cancer and the leading cause of cancer-related death worldwide. ${ }^{1}$ In 2015, lung cancer ranked first for cancer-related morbidity and mortality, with approximately 733000 new cases and 610200 deaths recorded. ${ }^{2}$ Chemotherapy is an important component of systematic therapy for patients with advanced disease. ${ }^{3}$ Early prediction of treatment response and survival is paramount 
for these individuals, allowing clinicians and patients to make decisions according to the patient-specific prognosis. Along with demographic and clinical characteristics, patient-reported outcomes, mainly health-related quality of life (HRQoL), have emerged as prognostic factors. $^{4}$

HRQoL is a subjective, personal, multidimensional, patient-reported outcome assessed according to physical, psychosocial and functional well-being (FWB).$^{5}$ Studies have suggested that pretreatment HRQoL and HRQoL at baseline were significantly associated with clinical benefits in patients with lung cancer. ${ }^{6-8}$ A meta-analysis involving over 10000 patients with cancer revealed that high baseline HRQoL was a prognostic factor for longer overall survival (OS). ${ }^{9}$ Studies investigating the relationship between baseline HRQoL and prognosis have mainly been conducted in countries in America and Europe. To the best of our knowledge, only two studies have evaluated this relationship in Chinese patients with lung cancer. Fielding and Wong found that baseline physical well-being could predict OS in patients with lung cancer in Hong Kong, while a study conducted in Taiwan reported that baseline symptoms were associated with a higher risk of death. ${ }^{10}{ }^{11}$ Consequently, more evidence is needed to deepen our understanding of the relationship between baseline HRQoL and clinical outcomes in Chinese patients with lung cancer.

Besides the measurement of HRQoL at one time point, studies have also focused on the prognostic value of changes in HRQoL in patients with lung cancer; however, the obtained results have been inconsistent. ${ }^{12}$ Cella $e t$ al analysed secondary data from clinical trials and suggested that improvements in HRQoL from baseline to 12 weeks could predict response to treatment and time to progression in patients with lung cancer. ${ }^{13}$ Another study examined data from the European Organization for Research on Treatment of Cancer 08975 lung cancer trial and reported that increased social function from baseline to completion of cycle 2 of chemotherapy was associated with a $9 \%$ lower risk of death. ${ }^{14}$ Meanwhile, a prospective study in Sweden also indicated that a decline in HRQoL from baseline to 6 months was associated with a 13\%-18\% higher risk of death among patients who underwent lung cancer surgery. ${ }^{15}$ In contrast, Gupta et al followed 430 patients with advanced lung cancer and found that a change in HRQoL from baseline to 3 months was not predictive of survival. ${ }^{16}$ Similarly, Mizutani et al indicated that symptom improvement from baseline to cycle 2 completion was not associated with favourable OS. ${ }^{17}$ These inconsistent findings pose the dilemma among clinicians and patients alike as to whether changes in HRQoL can indeed predict prognosis. Moreover, most studies investigating changes in HRQoL over recent years have mainly focused on measurements from baseline to 6 weeks or later, and whether earlier changes in HRQoL occur during antitumour therapy, and whether they have predictive potential, has remained largely ignored. Consequently, in this study, we explored the prognostic effects of early changes in HRQoL from baseline to completion of cycle 1 of chemotherapy.

Large variability in HRQoL measures has been observed in previous studies, ${ }^{18}{ }^{19}$ highlighting the need to select sensitive measures that reflect HRQoL. ${ }^{20}$ The Functional Assessment of Cancer Therapy-Lung (FACT-L) is a widely used, disease-specific measure of HRQoL for patients with lung cancer. ${ }^{21}{ }^{22}$ The Lung Cancer Subscale (LCS) of FACT-L and Trial Outcome Index (TOI), which consist of Physical Well-being Subscale, Functional Well-being Subscale and LCS, are suggested as sensitive measures to detect the relationship between HRQoL and prognosis. ${ }^{13}$ However, to our knowledge, a comparative analysis between TOI and LCS has not been undertaken to date, and it remains unknown which of these measures has higher sensitivity and clinical feasibility.

In the current study, we aimed to (1) investigate whether baseline HRQoL could predict response to treatment and survival in Chinese patients with advanced lung cancer; (2) investigate whether early changes in HRQoL from baseline to cycle 1 completion could predict response to treatment and survival; and (3) compare the prognostic value of TOI and LCS and thereby identify an appropriate prognostic indicator with high sensitivity and feasibility.

\section{METHODS}

\section{Design, setting and patients}

This study adopted a prospective observational design, collecting patient survey data and electronic health record data from the Medical Oncological unit of the Sun Yat-sen University Cancer Center. Convenience sampling was used for the selection of eligible patients from November 2012 to January 2015. Patients were included if they were newly diagnosed with primary lung cancer, 18-75 years old, chemo-naive and ambulatory (scored $0-1$ on the Eastern Cooperative Oncology Group (ECOG) performance status rating). Patients were excluded if they had previously received any antitumour treatment when contacted to participate in the research; were diagnosed as schizophraenia, mood disorders (eg, depressive disorder and bipolar disorder), disorders of consciousness (eg, drowsiness, clouding of consciousness, sopor, coma or delirium) or dementia; or were pregnant or lactating.

\section{Variables and measures}

HRQoL

FACT-L V.4.0 was used to assess HRQoL. ${ }^{21} 22$ The FACT-L is widely used in international studies and has been established as a reliable and validated tool. ${ }^{23}$ The FACT-L is a 36-item scale, including four general subscales (physical well-being (PWB), FWB, social/family well-being and emotional well-being) and a 7 -item LCS. ${ }^{21}$ The FACT-L is a 5-point Likert scale, ranging from $0=n o t$ at all to $4=$ verymuch. The positively stated items were scored directly from 0 to 4 , while the negatively stated items were scored in reverse. The score for each subscale was 
calculated by adding up all the item scores within the subscale. ${ }^{21}$ The Chinese version of the FACT-L has acceptable construct validity, discriminative validity, criterionrelated validity and internal reliability. ${ }^{23}$ In our study, Cronbach's $\alpha$ coefficient of FACT-L was $0.612,0.795$ and 0.827 for LCS, PWB and FWB, respectively.

Studies have suggested that the TOI and LCS are the most relevant indicators of quality of life for patients with lung cancer. ${ }^{21}$ The TOI was derived by adding the PWB, FWB and LCS scores. The possible score range was 0-84 for the TOI and 0-28 for the LCS. Higher TOI or LCS scores indicated better patient-perceived quality of life or fewer symptoms. The cut-off points for baseline TOI and LCS were determined according to median baseline TOI and LCS scores, as previously reported. ${ }^{17}$ Early changes in HRQoL were calculated using TOI/LCS at 3 weeks (ie, first cycle completed) minus TOI/LCS at baseline. For the TOI, changes $\leq-5,-4$ to 4 and $\geq 5$ were categorised as deteriorated, unchanged and improved, respectively. ${ }^{13}$ For the LCS, changes $\leq-2,-1$ to 1 and $\geq 2$ were, respectively, categorised as deteriorated, unchanged and improved. ${ }^{13}$

\section{Prognosis}

Prognosis was measured with respect to objective response to treatment at second cycle of chemotherapy completed and OS at 5 years. To evaluate response to treatment, tumour sizes at baseline were compared with those at completion of the second cycle of chemotherapy. Both CT and MRI were used to measure tumour shrinkage; however, each participant used only one examination method for comparison. Response to treatment was categorised as complete response (CR), partial response (PR), stable disease (SD) or progressive disease (PD) as per the Response Evaluation Criteria in Solid Tumors V.1.1. ${ }^{24}$ OS was defined as the period from diagnosis to the date of death from any cause or last follow-up.

\section{Demographic and clinical characteristics}

Each patient's age, gender, diagnosis, cancer stage, histological subtype, type of treatment, ECOG performance status rating and smoking history were collected as covariates in the analysis.

\section{Procedure}

Trained researchers and research doctors were responsible for contacting eligible patients in the Medical Oncological unit, obtaining informed consent, and delivering and collecting surveys. Patients were invited to participate voluntarily. All patients were assured that refusal to participate would not affect their future treatment. Participants could withdraw from the study at any time. Patient survey data were collected at baseline (at diagnosis, before chemotherapy) and at 3 weeks (at the end of the first cycle of chemotherapy). The patients were asked to fill the questionnaire using the cloud QoL system. ${ }^{25}$ The patients were followed regularly by the hospital's follow-up team after the completion of treatment. If a patient died during the follow-up, the family caregivers were asked to report the date of death. To achieve long-term prognosis, the patients were followed for 5 years. Survival at 5 years is a prognostic indicator widely used in patients with lung cancer. ${ }^{26}$ Thus, the last follow-up was September 2019. During the follow-up, 183 deaths $(75.3 \%)$ occurred among the 243 enrolled patients, which suggested data maturity (ie, the number of participants at risk is unduly small) ${ }^{27}$

\section{Data analysis}

Descriptive statistics were used to depict patient's demographic and clinical characteristics, quality of life, response to treatment and OS. A $\chi^{2}$ analysis was used to examine the distribution of differences in responses to treatment among patients with different baseline HRQoL and early changes in HRQoL. Multivariate logistic regression modelling was used to estimate the relationship among HRQoL at baseline, early changes in HRQoL and response to treatment in patients with lung cancer after controlling for demographic and clinical characteristics. Kaplan-Meier survival analysis and Cox proportional hazard models were used to determine whether baseline HRQoL and early changes in HRQoL were independent prognostic indicators of survival. In addition, the receiver operating characteristics analysis was performed and area under curve (AUC) values were compared with evaluate the differences in the predictive ability of each HRQoL indicator. To avoid collinearity, each HRQoL variable was analysed separately in multivariate analyses. Cases with missing data were excluded pairwise. A 0.05 level of significance was set for all statistical analyses. All analyses were conducted using SPSS V.24.0 (SPSS).

\section{Public and patient involvement}

No patient was involved in the study design, data collection, data analysis or interpretation of the results.

\section{RESULTS}

Demographic and clinical characteristics of the patients

During the data collection period, 375 eligible patients were approached, 314 of which consented to participate in the study at baseline, while 71 refused to fill in the questionnaire after the completion of cycle 1 of chemotherapy (see online supplemental figure 1). As a result, a total of 243 patients completed two rounds of surveys were included in the study. The average age of the patients was 56.5 years at baseline. Most of the patients were man $(77.0 \%)$ and half had a history of smoking $(53.1 \%)$. More than three-quarters of the patients had stage IV cancer $(84.4 \%)$ at diagnosis and the histological subtype was predominately adenocarcinoma $(62.6 \%)$. The treatment regimen for most of the patients $(62.6 \%)$ was platinum and pemetrexed. The ECOG performance status rating was 0 in $93.8 \%$ of the patients (table 1 ).

\section{Description of HRQOL, response to treatment and OS}

The average TOI score was 53.1 \pm 9.9 (range: $0-84$ ) and the average LCS score was 20.4 \pm 3.8 (range: $0-28$ ) at 
Table 1 Demographic and clinical characteristics, healthrelated quality of life, response to treatment and overall survival in patients with lung cancer $(\mathrm{N}=243)$

\begin{tabular}{lc|}
\hline Variables & $\mathbf{n}(\%) /$ Mean \pm SD/median $\left(\mathbf{P}_{25} \mathbf{P}^{-}\right.$ \\
\hline Gender (male) & $187(77.0)$ \\
\hline Age (yrs) & $56.5 \pm 10.2$ \\
\hline Smokers & $129(53.1)$ \\
\hline Histological subtype & \\
\hline Adenocarcinoma & $157(64.6)$ \\
\hline Squamous carcinoma & $35(14.4)$ \\
\hline Small cell carcinoma & $41(16.9)$ \\
\hline Other & $10(4.1)$ \\
\hline Clinical stage & \\
\hline Illb & $38(15.6)$ \\
\hline IV & $205(84.4)$ \\
\hline Treatment arm & \\
\hline Platinum+pemetrexed & $152(62.6)$ \\
\hline Platinum+etoposide & $44(18.1)$ \\
\hline Platinum+paclitaxel & $32(13.2)$ \\
\hline Platinum+gemcitabine & $7(2.9)$ \\
\hline Other & $8(3.2)$ \\
\hline
\end{tabular}

Baseline performance status (ECOG)

\begin{tabular}{|c|c|}
\hline 1 (some symptoms, but ambulatory) & $15(6.2)$ \\
\hline 0 (normal activity) & 227 (93.8) \\
\hline TOI at baseline ${ }^{*}$ & $53.1 \pm 9.9$ \\
\hline LCS at baseline ${ }^{\star}$ & $20.4 \pm 3.8$ \\
\hline TOI at 3 weeks & $54.0 \pm 10.2$ \\
\hline LCS at 3 weeks & $21.6 \pm 3.4$ \\
\hline \multicolumn{2}{|l|}{ Changes of TOI $\dagger$} \\
\hline Improved & $88(36.2)$ \\
\hline No change & $77(31.7)$ \\
\hline Declined & $78(32.1)$ \\
\hline \multicolumn{2}{|l|}{ Changes of LCS $†$} \\
\hline Improved & $109(44.9)$ \\
\hline No change & 80 (32.9) \\
\hline Declined & $54(22.2)$ \\
\hline \multicolumn{2}{|l|}{ Response to treatment } \\
\hline $\mathrm{CR}$ & $0(0)$ \\
\hline PR & $101(41.6)$ \\
\hline SD & $115(47.3)$ \\
\hline PD & $27(11.1)$ \\
\hline \multicolumn{2}{|l|}{ Vital status at the last follow-up } \\
\hline Died & $183(75.3)$ \\
\hline Alive & $14(5.8)$ \\
\hline Unknown & $46(18.9)$ \\
\hline Overall survival (months) & $17(7-26)$ \\
\hline
\end{tabular}

${ }^{*}$ The median for Trial Outcome Index (TOI) at baseline and Lung Cancer Subscale (LCS) at baseline was 54 and 21, respectively.

tChanges in TOI were defined as 'improved'=improved 5 points or greater, 'unchanged'=changed within -4 to 4 points, 'declined'=declined 5 points or greater; changes in LCS were defined as improved=improved 2 points or greater, unchanged=changed within -1 to 1 points, declined=declined 2 points or greater. CR, complete remission; ECOG, Eastern Cooperative Oncology Group; PD, progressive disease; $\mathrm{PR}$, partial remission; $\mathrm{SD}$, stable disease.
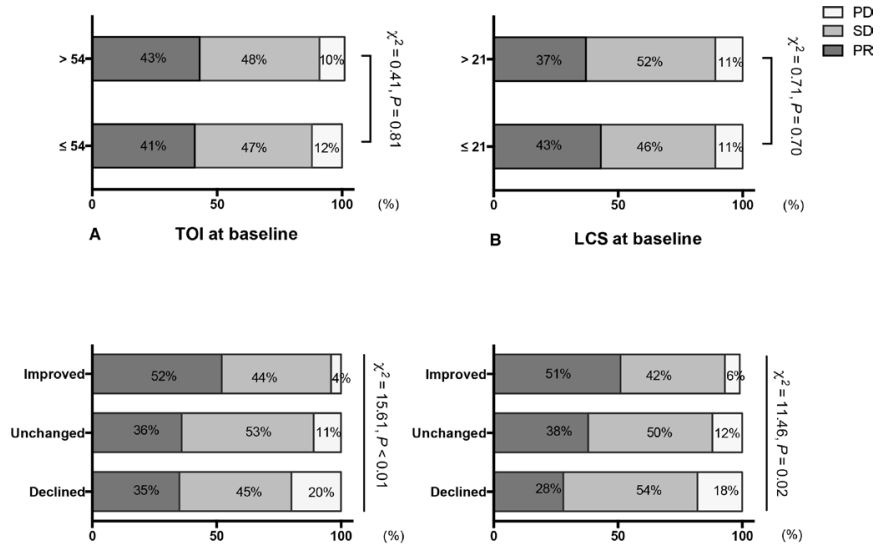

C. Changes in TO

D Changes in LCS

Figure 1 Univariate analysis of associations between health-related quality of life and treatment response. LCS, Lung Cancer Subscale; PR, partial response; PD, progressive disease; SD, stable disease; TOI, Trial Outcome Index.

baseline for the patients with lung cancer. The TOI score improved by at least 5 points for approximately one-third of the patients $(\mathrm{n}=88,36.2 \%)$ and declined for $32.1 \%$ of them $(n=78)$. For the LCS, approximately half of the patients showed an improvement of 2 points or more from baseline to 3 weeks $(n=109,44.9 \%)$, while $22.2 \%$ exhibited a decline $(\mathrm{n}=54)$ (table 1$)$.

As shown in table 1 , none of the patients showed CR at 6 weeks, with most showing SD $(47.3 \%)$ or PR $(41.6 \%)$, while approximately $10 \%$ showed PD $(11.1 \%)$. The median OS time was 17 months. At the last follow-up, 183 patients had died $(75.3 \%), 14$ were alive $(5.8 \%)$ and 46 were lost to follow-up (18.9\%).

\section{Associations between baseline $\mathrm{HRQ} \mathrm{OL}$ and prognosis}

We divided HRQoL at baseline into categories according to the median TOI and LCS values. The results of $\chi^{2}$ tests showed that the distribution of responses to treatment did not vary significantly among patients with different baseline HRQoL ( $>0.05$ ) (figure 1). After controlling for patient's characteristics, the logistic regression analysis showed that the baseline HRQoL was not associated with responses to treatment $(p>0.05)$ (figure 2). In the Kaplan-Meier survival analysis, OS was significantly longer for patients with high TOI or LCS values at baseline than for those with low scores (TOI: 19 vs 14 months, $p=0.02$; LCS: 19 vs 14 months, $p=0.03$ ) (figure 3). The results from multivariate Cox hazard regression modelling also showed that patients with lower TOI $(80.0 \%$ vs $69.9 \%$, adjusted HR (aHR) $=1.36,95 \%$ CI 1.01 to 1.84 ) or LCS ( $77.6 \%$ vs $72.5 \%$, aHR $=1.36,95 \%$ CI 1.01 to 1.83 ) values at baseline were at higher risk for death after controlling for demographic and clinical factors (table 2). Among the two HRQoL indicators, LCS displayed a higher AUC value (0.592, $95 \%$ CI 0.516 to 0.667$)$ than TOI $(\mathrm{AUC}=0.578$, $95 \%$ CI 0.503 to 0.653 ) at baseline (figure 4 ), and was, therefore, a better predictor of OS. 


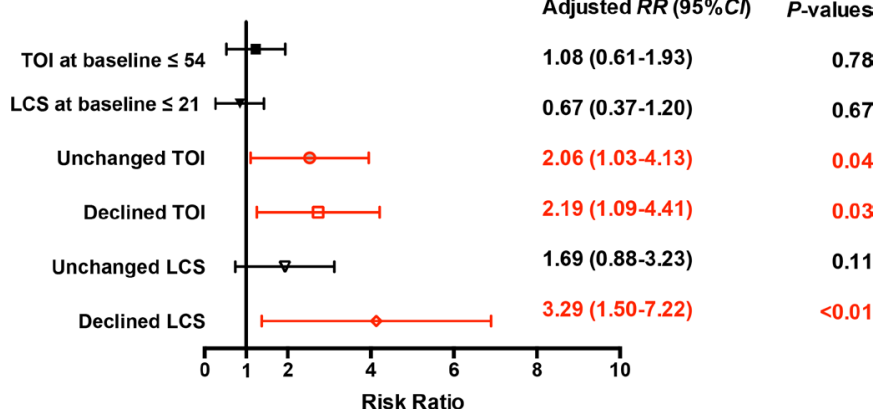

Figure 2 Multivariate analysis of associations between health-related quality of life (HRQoL) and treatment response. Relationships between HRQoL and treatment response $(n=243)$. Note: the incidence in the multivariate logistic regression models was defined as 'stable disease or progressive disease'. Age at diagnosis, gender, histological subtype, clinical stage, treatment arm, Eastern Cooperative Oncology Group performance status and smoking history were controlled in all multivariate logistic regression models. $P$ values for Hosmer and Lemeshow Test were $>0.05$ for all models. LCS, Lung Cancer Subscale; TOI, Trial Outcome Index.

\section{Associations between changes in HRQoL and prognosis}

The greatest proportion of PR was seen among patients with improved TOI or LCS scores from baseline to 3 weeks $(\mathrm{p}<0.05)$, as shown in figure 1 . The logistic regression modelling suggested that patients with lower TOI $(65 \%$ vs $48 \%$, adjusted risk ratio $(\mathrm{aRR})=2.19,95 \%$ CI 1.09 to 4.41$)$, unchanged TOI ( $64 \%$ vs $48 \%$, aRR $=2.06,95 \%$ CI 1.03 to 4.13 ) and lower LCS ( $72 \%$ vs $48 \%$, aRR $=3.29,95 \%$ CI 1.50 to 7.22 ) values were more likely to show SD or PD (figure 2). Early changes in LCS scores from baseline to 3 weeks were associated with a higher AUC value $(0.609$, $95 \%$ CI 0.542 to 0.677 ) compared with early changes in TOI scores $(0.582,95 \%$ CI 0.514 to 0.650$)$ (figure 4 ). However, changes in TOI and LCS scores were not associated with OS or risk for death after controlling for demographic and clinical factors and baseline HRQoL (figure 3; table 2).

\section{DISCUSSION}

In this study, we established the first prospective cohort of 243 Chinese patients with advanced lung cancer to determine the prognostic value of early changes in HRQoL. Drawing on this cohort, our study revealed that lower baseline HRQoL was associated with poorer OS, while an early deterioration in HRQoL was associated with a less favourable response to chemotherapy.
A

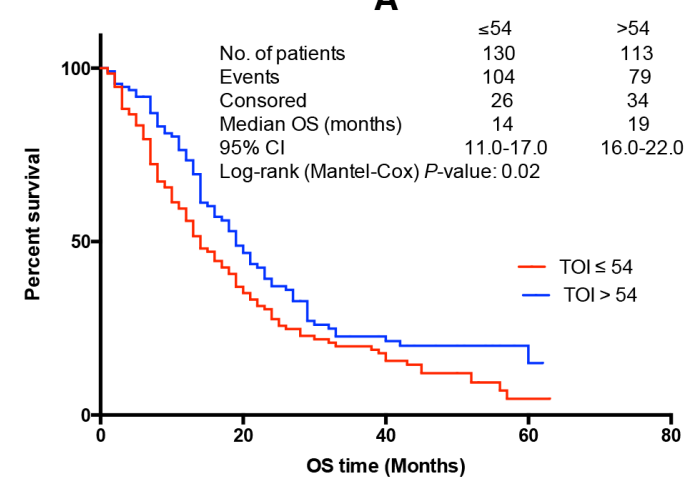

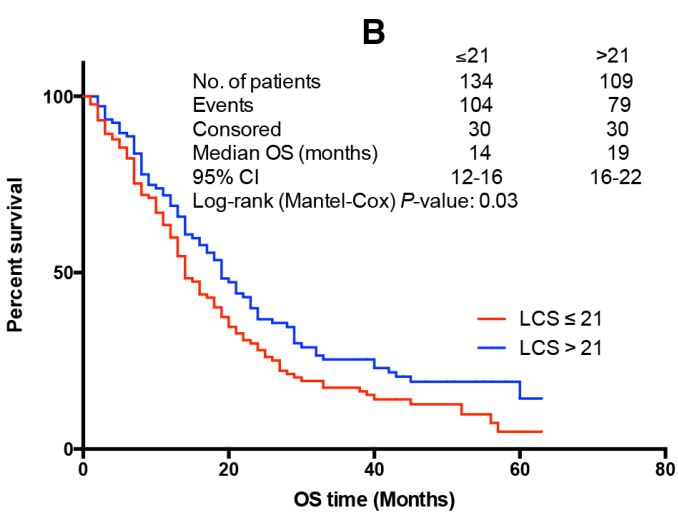

Number at risk

$\begin{array}{lllll}\leq 21 & 134 & 37 & 11 & 0 \\ >21 & 109 & 45 & 19 & 3\end{array}$

\section{C}

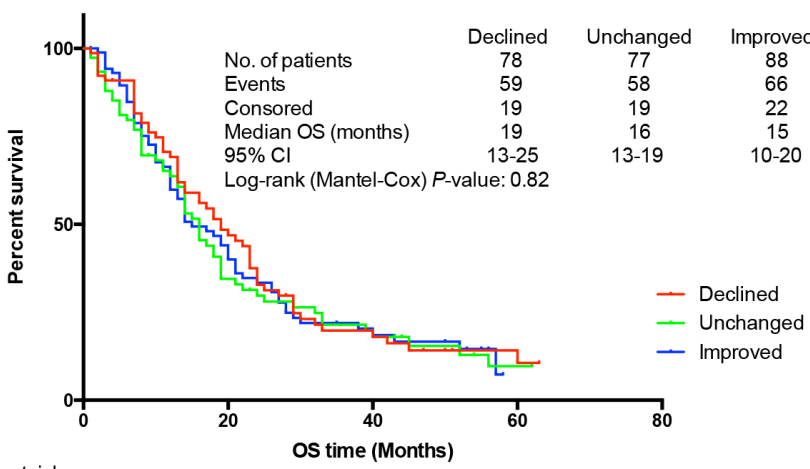

Number at risk Declined 78 Unchanged 77 Improved

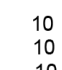

10
10

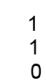

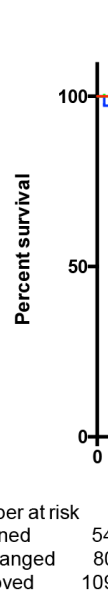
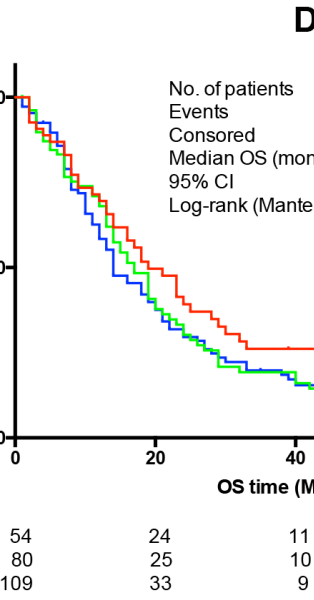

D

Figure 3 Kaplan-Meier overall survival (OS) estimates according to (A) baseline Trial Outcome Index (TOI), (B) baseline Lung Cancer Subscale (LCS), (C) early changes in TOI and (D) early changes in LCS. 
Table 2 Univariate and multivariate Cox proportional hazards models of health-related quality of life $(n=243)$

\begin{tabular}{|c|c|c|c|c|}
\hline \multirow[b]{2}{*}{ Factors } & \multicolumn{2}{|l|}{ Univariate } & \multicolumn{2}{|l|}{ Multivariate } \\
\hline & Hazard ratio $(95 \% \mathrm{Cl})$ & $P$ value & Hazard ratio $(95 \% \mathrm{Cl})$ & $P$ value \\
\hline \multicolumn{5}{|l|}{ TOI at baseline } \\
\hline \multicolumn{5}{|l|}{$>54$ (ref.) } \\
\hline \multicolumn{5}{|l|}{ LCS at baseline } \\
\hline \multicolumn{5}{|l|}{$>21$ (ref.) } \\
\hline \multicolumn{5}{|c|}{ Changes in $\mathrm{TOI}$} \\
\hline \multicolumn{5}{|c|}{ Improved (ref.) } \\
\hline Unchanged & 1.05 (0.65 to 1.32$)$ & 0.93 & $1.07(0.74$ to 1.54$)$ & 0.72 \\
\hline Declined & 0.93 (0.65 to 1.32$)$ & 0.81 & 0.96 (0.67 to 1.39$)$ & 0.85 \\
\hline \multicolumn{5}{|c|}{ Improved (ref.) } \\
\hline Unchanged & 0.91 (0.66 to 1.28$)$ & 0.60 & $0.91(0.64$ to 1.28$)$ & 0.59 \\
\hline Declined & 0.72 (0.49 to 1.06$)$ & 0.10 & $0.68(0.45$ to 1.01$)$ & 0.05 \\
\hline
\end{tabular}

Note: age at diagnosis, gender, histological subtype, clinical stage, treatment arm, Eastern Cooperative Oncology Group performance status rating and smoking history were controlled in all multivariate Cox proportional hazards models. ${ }^{*} \mathrm{P}<0.05$.

LCS, Lung Cancer Subscale; ref, reference; TOI, Trial Outcome Index.

\section{Baseline HRQoL and prognosis}

Our results showed that scores equal to or less than 54 and 21 for the TOI and LCS at baseline, respectively, could predict low OS. These findings corroborate those of previous studies conducted in the USA and Europe showing that baseline HRQoL can serve as a prognostic indicator. ${ }^{28} 29$ These observations imply that patients with low baseline HRQoL may be more fragile and vulnerable, and may be at higher risks for financial difficulties, and were thus less likely to receive adequate antitumour treatment. ${ }^{1730}$ Nevertheless, we could not establish associations between baseline HRQoL and response to treatment. This is inconsistent with a previous study that reported positive associations between pretreatment HRQoL and response to chemotherapy. ${ }^{31}$ More evidence is needed to delineate the mechanism underlying how pretreatment

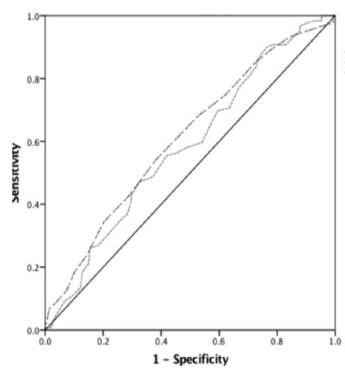

A Changes in HRQOL predicting OS

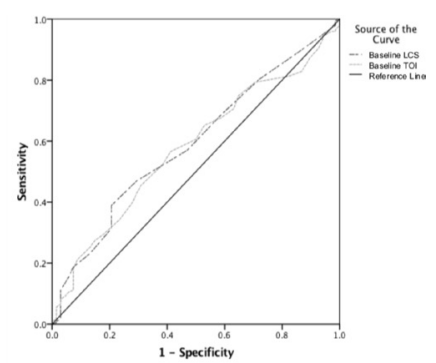

B Baseline HRQOL predicting response to
Figure 4 Area under curves for the ability of early changes in health-related quality of life (HRQOL) and baseline HRQoL to predict overall survival and response to chemotherapy. LCS, Lung Cancer Subscale; TOI, Trial Outcome Index.
HRQoL affects response to treatment. Overall, our findings are consistent with those of previous studies, and highlight the profound influence of baseline HRQoL on long-term prognosis. Our sample of patients with lung cancer was broadly representative of patients in the real world, and we suggest that scores of 54 for TOI and 21 for LCS could be used as thresholds to identify Chinese patients with lung cancer receiving chemotherapy who are at risk of poor OS.

\section{Early change in $\mathrm{HRQ} \mathrm{OL}$ and prognosis}

Previous studies have suggested that 5-point and 2-point changes in TOI and LCS scores from baseline to completion of the second cycle of chemotherapy are clinically meaningful. ${ }^{13}{ }^{31}$ Here, we have identified even earlier outcome predictors, with our results indicating that a 5-point or 2-point decline in TOI or LCS scores from baseline to the completion of cycle 1 may imply SD or PD. Moreover, our findings suggest that an earlier change in HRQoL may reflect early tumour burden changes, thereby providing hints for treatment responses. Earlier response evaluation is recommended for patients with reduced HRQoL to allow treatment to be adjusted accordingly.

\section{Comparison between TOI and LCS}

In our study, baseline LCS scores and early changes in LCS scores may be the most appropriate measures for predicting OS and initial response to treatment, respectively. As the LCS has only 7 items and can provide valuable prognostic information, LCS data should be 
collected routinely within clinical settings to allow the identification of patients at high risks of poor prognosis.

\section{Strengths and limitations}

This is the first cohort established to explore the relationship between early changes in HRQoL and prognosis in patients with advanced lung cancer in China. Given the real-world characteristics of these data, our results might be more precise than previous findings obtained through the analysis of secondary data from clinical trials. In addition, this study represents one of the first attempts to identify clinically meaningful changes in HRQoL. The changes in HRQoL occurring between baseline and the completion of the first cycle of chemotherapy could be detected earlier than those reported in other studies, and also had acceptable sensitivity. Although treatments are consistently being updated, the present findings are beneficial for decision-making for both clinicians and patients with advanced lung cancer receiving chemotherapy.

Despite the above observations, this study had several limitations. First, the study was conducted in a single centre using a convenience sampling method, which cannot represent all patients with lung cancer in China. Second, only patients receiving chemotherapy were included, thus excluding those who underwent surgery or received radiotherapy. Third, to avoid heavy measurement burden for patients, HRQoL measurement included only PWB, FWB and lung cancer-specific symptom burden, while psychological and social well-being were not considered. Fourth, although FACT-L is widely used in international studies, the Cronbach's $\alpha$ of LCS was relatively low in this study, which may indicate a low internal consistency. Fifth, gene mutations were not included in the study, as this information was not available for more than onethird of the patients. Finally, approximately one-third of patients were lost to follow-up, which may have biased the survival analysis.

\section{CONCLUSIONS}

Overall, our study demonstrated that pretreatment HRQoL assessment and early changes in HRQoL have predictive value for prognosis in patients with lung cancer. An LCS score of $\leq 22$ at baseline and a $\geq 2$-point decline in the LCS score at the end of the first cycle of chemotherapy are suggested as suitable cutoffs for the identification of patients at high risk for poor prognosis and the detection of early treatment effects, thereby helping clinicians determine the best treatment strategy for each patient with lung cancer.

\section{Author affiliations}

${ }^{1}$ Department of Nursing, State Key Laboratory of Oncology in South China, Collaborative Innovation Center for Cancer Medicine, Sun Yat-sen University Cancer Center, Guangzhou, China

${ }^{2}$ Department of Clinical Research, State Key Laboratory of Oncology in South China, Collaborative Innovation Center for Cancer Medicine, Sun Yat-sen University Cancer Center, Guangzhou, China
${ }^{3}$ Department of Medical oncology, State Key Laboratory of Oncology in South China, Collaborative Innovation Center for Cancer Medicine, Sun Yat-sen University Cancer Center, Guangzhou, Guangdong, China

Contributors JL: conceptualisation, study design, data collection, data analysis, writing-original draft, writing-revision and editing, visualisation, funding acquisition YM: conceptualisation, study design, data collection, data analysis, writing-revision and editing. RG: conceptualisation, study design, data collection, writing-revision and editing. XL, YW, JY, JZ: study design, data collection, writing-revision and editing. YH: conceptualisation, study design, data collection, writing-revision and editing, supervision, funding acquisition. $\mathrm{HQ}$ : conceptualisation, study design, data collection, writing-revision and editing, supervision, project administration. LZ: guarantor for the study.

Funding This work was supported by the Science and Technology Program of Guangzhou (NO. 201704020072); the Medical Scientific Research Foundation of Guangdong Province (NO. C2018062); the Humanities and Social Science Program of Ministry of Education of China (NO. 2OYJCZHO94).

Competing interests None declared.

Patient and public involvement Patients and/or the public were not involved in the design, or conduct, or reporting, or dissemination plans of this research.

Patient consent for publication Not applicable.

Ethics approval The study was approved by the Ethics Committee of Sun Yat-sen University Cancer Center (ID for the approval: 2012043). Participants gave informed consent to participate in the study before taking part.

Provenance and peer review Not commissioned; externally peer reviewed. Data availability statement Data are available upon reasonable request.

Open access This is an open access article distributed in accordance with the Creative Commons Attribution Non Commercial (CC BY-NC 4.0) license, which permits others to distribute, remix, adapt, build upon this work non-commercially, and license their derivative works on different terms, provided the original work is properly cited, appropriate credit is given, any changes made indicated, and the use is non-commercial. See: http://creativecommons.org/licenses/by-nc/4.0/.

\section{ORCID iD}

Jiali Liu http://orcid.org/0000-0001-7202-0623

\section{REFERENCES}

1 International Agency for Research on Cancer. World cancer report: cancer research for cancer prevention. Available: https://publications. iarc.fr/586 [Accessed 24 Jul 2021].

2 Chen W, Zheng R, Baade PD, et al. Cancer statistics in China, 2015. CA Cancer J Clin 2016;66:115-32.

3 Arbour KC, Riely GJ, Arbour Kathryn C. Systemic therapy for locally advanced and metastatic non-small cell lung cancer: a review. JAMA 2019;322:764-74.

4 Bouazza YB, Chiairi I, El Kharbouchi O, et al. Patient-Reported outcome measures (PROMs) in the management of lung cancer: a systematic review. Lung Cancer 2017;113:140-51.

5 Polanski J, Jankowska-Polanska B, Rosinczuk J, et al. Quality of life of patients with lung cancer. Onco Targets Ther 20162016;9:1023-8.

6 Mierzynska J, Piccinin C, Pe M, et al. Prognostic value of patientreported outcomes from international randomised clinical trials on cancer: a systematic review. Lancet Oncol 2019;20:e685-98.

7 Pinheiro LC, Reeve BB. Investigating the prognostic ability of healthrelated quality of life on survival: a prospective cohort study of adults with lung cancer. Support Care Cancer 2018;26:3925-32.

8 Fiteni F, Vernerey D, Bonnetain F, et al. Prognostic value of healthrelated quality of life for overall survival in elderly non-small-cell lung cancer patients. Eur J Cancer 2016;52:120-8.

9 Quinten C, Coens C, Mauer M, et al. Baseline quality of life as a prognostic indicator of survival: a meta-analysis of individual patient data from EORTC clinical trials. Lancet Oncol 2009;10:865-71.

10 Fielding R, Wong WS. Quality of life as a predictor of cancer survival among Chinese liver and lung cancer patients. Eur J Cancer 2007;43:1723-30.

11 Li T-C, Li C-I, Tseng C-H, et al. Quality of life predicts survival in patients with non-small cell lung cancer. BMC Public Health 2012;12:790.

12 Eton DT, Fairclough DL, Cella D, et al. Early change in patientreported health during lung cancer chemotherapy predicts clinical 
outcomes beyond those predicted by baseline report: results from eastern cooperative Oncology Group study 5592. J Clin Oncol 2003;21:1536-43.

13 Cella D, Herbst RS, Lynch TJ, et al. Clinically meaningful improvement in symptoms and quality of life for patients with nonsmall-cell lung cancer receiving gefitinib in a randomized controlled trial. J Clin Oncol 2005;23:2946-54.

14 Ediebah DE, Coens C, Zikos E, et al. Does change in health-related quality of life score predict survival? analysis of EORTC 08975 lung cancer trial. Br J Cancer 2014;110:2427-33.

15 Möller A, Sartipy U. Associations between changes in quality of life and survival after lung cancer surgery. J Thorac Oncol 2012;7:183-7.

16 Gupta D, Braun DP, Staren ED. Association between changes in quality of life scores and survival in non-small cell lung cancer patients. Eur J Cancer Care 2012;21:614-22.

17 Mizutani T, Ando M, Mizusawa J, et al. Prognostic value of lung cancer Subscale in older patients with advanced non-small cell lung cancer: an integrated analysis of JCOG0207 and JCOG0803/ WJOG4307L (JCOG1414A). J Geriatr Oncol 2018;9:583-8.

18 Dharma-Wardene M, Au H-J, Hanson J, et al. Baseline FACT-G score is a predictor of survival for advanced lung cancer. Qual Life Res 2014;13:1209-16.

19 Grande GE, Farquhar MC, Barclay SIG, et al. Quality of life measures (EORTC QLQ-C30 and SF-36) as predictors of survival in palliative colorectal and lung cancer patients. Palliat Support Care 2009;7:289-97.

20 Eser S, Göksel T, Erbaycu AE, et al. Comparison of generic and lung cancer-specific quality of life instruments for predictive ability of survival in patients with advanced lung cancer. Springerplus 2016;5:5.

21 Cella DF, Bonomi AE, Lloyd SR, et al. Reliability and validity of the functional assessment of cancer Therapy-Lung (FACT-L) quality of life instrument. Lung Cancer 1995;12:199-220.

22 American Thoracic Society,. Functional Assessment of Cancer Therapy - Lung (FACT-L), 1999. Available: https://qol.thoracic.org/ sections/instruments/fj/pages/fact-I.html [Accessed 28 May 2020].
23 Wan C, Zhang C, Cai L, et al. Psychometric properties of the Chinese version of the FACT-L for measuring quality of life in patients with lung cancer. Lung Cancer 2007;56:415-21.

24 Eisenhauer EA, Therasse P, Bogaerts J, et al. New response evaluation criteria in solid tumours: revised RECIST guideline (version 1.1). Eur J Cancer 2009;45:228-47.

25 Ma Y, Yang Y, Huang Y, et al. An investigation of symptom burden and quality of life in Chinese chemo-naïve advanced lung cancer patients by using the Instrument-Cloud QOL system. Lung Cancer 2014;84:301-6.

26 Grivaux M, Zureik M, Marsal L, et al. Five-Year survival for lung cancer patients managed in general hospitals. Rev Mal Respir 2011;28:e31-8.

27 Rich JT, Neely JG, Paniello RC, et al. A practical guide to understanding Kaplan-Meier curves. Otolaryngol Head Neck Surg 2010;143:331-6.

28 Maione P, Perrone F, Gallo C, et al. Pretreatment quality of life and functional status assessment significantly predict survival of elderly patients with advanced non-small-cell lung cancer receiving chemotherapy: a prognostic analysis of the multicenter Italian lung cancer in the elderly study. J Clin Oncol 2005;23:6865-72.

29 Qi Y, Schild SE, Mandrekar SJ, et al. Pretreatment quality of life is an independent prognostic factor for overall survival in patients with advanced stage non-small cell lung cancer. J Thorac Oncol 2009;4:1075-82.

30 Thronicke A, von Trott P, Kröz M, et al. Health-Related quality of life in patients with lung cancer applying integrative oncology concepts in a certified cancer centre. Evid Based Complement Alternat Med 2020;2020:1-9.

31 Cella D, Eton DT, Fairclough DL, et al. What is a clinically meaningful change on the functional assessment of cancer Therapy-Lung (FACT-L) questionnaire? results from eastern cooperative Oncology Group (ECoG) study 5592. J Clin Epidemiol 2002;55:285-95. 